\title{
AAV-Mediated Astrocyte-Specific Gene Expression under Human ALDH1L1 Promoter in Mouse Thalamus
}

\author{
Wuhyun Koh ${ }^{1,2}$, Yongmin Mason Park ${ }^{1,2}$, Seung Eun Lee ${ }^{3 *}$ and C. Justin Lee ${ }^{1,2,4 *}$ \\ ${ }^{1}$ Division of Bio-Medical Science \& Technology, Department of Neuroscience, KIST School, Korea University of Science and \\ Technology, Seoul 02792, ${ }^{2}$ Center for Neuroscience and Functional Connectomics, Korea Institute of Science and Technology \\ (KIST), Seoul 02792, ${ }^{3}$ Virus Facility, Research Animal Resource Center, Korea Institute of Science and Technology (KIST), \\ Seoul 02792, ${ }^{4}$ Center for Glia-Neuron Interaction, Korea Institute of Science and Technology (KIST), Seoul 02792, Korea
}

\begin{abstract}
Adeno-associated virus (AAV)-mediated gene delivery has been proposed to be an essential tool of gene therapy for various brain diseases. Among several cell types in the brain, astrocyte has become a promising therapeutic target for brain diseases, as more and more contribution of astrocytes in pathophysiology has been revealed. Until now, genetically targeting astrocytes has been possible by utilizing the glial fibrillary acidic protein $(G F A P)$ promoter. In some brain areas including thalamus, however, the GFAP expression in astrocytes is reported to be low, making it difficult to genetically target astrocytes using GFAP promoter. To study the function of astrocytes in thalamus, which serves as a relay station, there is a great need for identifying an alternative astrocyte-specific promoter in thalamus. Recently, a new astrocyte-specific promoter of ALDH1L1 has been identified. However, it has not been examined in thalamus. Here we developed and characterized an AAV vector expressing Cre recombinase under the human ALDH1L1 promoter, AAV-hALDH1L1-Cre. To test the cell-type specific expression of AAV-hALDH1L1-Cre, AAV virus was injected into several brain regions of Ail4 (RCL-tdTomato) mouse, which reports Cre activity by tdTomato expression. In thalamus, we observed that tdTomato was found mostly in astrocytes $(91.71 \%)$, with minimal occurrence in neurons (2.67\%). In contrast, tdTomato signal was observed in both neurons and astrocytes of the amygdala (neuron: 68.13\%, astrocyte: $28.35 \%$ ) and hippocampus (neuron: $76.25 \%$, astrocyte: $18.00 \%$ ), which is consistent with the previous report showing neuronal gene expression under rat ALDH1L1 promoter. Unexpectedly, tdTomato was found mostly in neurons (91.98\%) with minimal occurrence in astrocytes (6.66\%) of the medial prefrontal cortex. In conclusion, $\mathrm{h} A L D H 1 L 1$ promoter shows astrocyte-specificity in thalamus and may prove to be useful for targeting thalamic astrocytes in mouse.
\end{abstract}

Key words: Astrocytes, Dependovirus, Ventral Thalamic Nuclei, ALDH1L1 protein, human, Cre recombinase, Glial Fibrillary Acidic Protein

\section{INTRODUCTION}

Received November 18, 2017, Revised December 6, 2017, Accepted December 8, 2017

* To whom correspondence should be addressed. C. Justin Lee, TEL: 82-2-958-6940, FAX: 82-2-958-6937 e-mail: cjl@kist.re.kr

Seung Eun Lee, TEL: 82-2-958-6959, FAX: 82-2-958-6937

e-mail: selee@kist.re.kr
In the central nervous system, the most abundant cell type is astrocyte, which has been revealed to have a number of functions, including modulation of synaptic transmission $[1,2]$, relay for nutrients to adjacent neuron [3], clearance of waste molecules [4]. To study functions of astrocyte in many brain regions, researchers
Copyright $\odot$ Experimental Neurobiology 2017. www.enjournal.org
This is an Open Access article distributed under the terms of the Creative Commons Attribution Non-Commercial License (http://creativecommons.org/licenses/by-nc/4.0) which permits unrestricted non-commercial use, distribution, and reproduction in any medium, provided the original work is properly cited. 
have been relying heavily on the conventional astrocyte-specific marker, glial fibrillary acidic protein (GFAP) and its promoter after the discovery of its specificity for astrocytes [5]. However, it also has been reported that GFAP expression is low in several brain areas, including thalamus [6]. GFAP also has been known to be expressed transiently in neural progenitor cells, which could lead to neuronal expression near neurogenic areas [7]. Therefore, utilizing GFAP may not be optimal in those brain areas. Moreover, there is a pressing need for an alternative astrocyte-specific marker to study astrocytic functions in those brain areas.

There are two superb examples of utilizing astrocyte-specific promoter to study and target astrocytes in the mouse brain. First one is the generation of transgenic mouse lines such as GFAPEGFP mouse [8] and GFAP-Cre/ERT2 mouse [9]. Although utilizing transgenic mouse is one of the best choices to target astrocytes, it could be very resource- and time-consuming when they need to be transferred or maintained. It is also difficult to extend the study to other species beyond mouse. Another example is to apply astrocyte specific promoter in virus applications such as in GFAP-GFP virus [10]. Virus containing astrocyte-specific promoter allows researchers to examine the effects of a gene of interest in local astrocytes in a specific brain area where the virus is injected. Moreover, virus is easy to apply to different species, such as mouse, marmoset, monkey or even human. As more and more contribution of astrocytes in pathophysiology of brain diseases has been revealed, astrocyte-specific targeting with virus would be critically essential as a potential therapeutic tool [11]. Among viruses, adeno-associated virus (AAV) has become very popular with many prominent advantages: AAV is able to infect both dividing and non-dividing cell types with less immunogenic effect [12]. Ultimately, AAV promises to be the best available option for gene therapy for brain diseases $[13,14]$.

As an alternative to GFAP promoter, aldehyde dehydrogenase family 1, member L1 (ALDH1L1) was recently identified as a new astrocyte-specific marker in the brain [15]. The promoter of ALDH1L1 should be useful in brain regions where GFAP expression is low. There has been attempts to characterize the ALDH1L1 promoter to produce astrocyte specific reporter mouse line [16] or astrocyte-specific Cre recombinase expressing mouse line for cell-type specific manipulation [17]. However, application of $A L D H 1 L 1$ promoter for virus has been lacking. Recently, the rat ALDH1L1 (rALDH1L1) promoter has been constructed and characterized in substantia nigra pars compacta, hippocampal dentate gyrus, and striatum, with disappointing and unexpected expression in neurons [18]. Due to this unexpected neuronal expression by the $\mathrm{r} A L D H 1 L 1$ promoter, it is necessary to construct and test $A L D H 1 L 1$ promoters from other species in hope to find an effective astrocyte-specific promoter for virus.

In this study, we constructed and characterized human $A L$ DH1L1 (hALDH1L1) promoter in AAV vector to investigate the degree of astrocyte-specific expression in the mouse brain. $A A V$ vector was constructed to express Cre recombinase under hALDH1L1 promoter. The packaged AAV virus was delivered into Ail 4 mouse, whose transgene can be cut by Cre recombinase, resulting in tdTomato expression. By examining the celltype specificity by analyzing the population of tdTomato positive (tdTomato+) cells in infected area, we demonstrate that most of thalamic ventrobasal astrocytes express tdTomato fluorescence by AAV-hALDH1L1-Cre virus with high specificity.

\section{MATERIALS AND METHODS}

\section{Bioinformatics and plasmid construction}

The hALDHIL1 gene locus was identified using the UCSC Genome Browser on Human Dec. 2013 (GRCh38/hg38) Assembly. The genomic sequence from Chromosome 3 q21.3 (ch3:126,180,366-126,181,669) including exons, introns and a 1.3 $\mathrm{kb}$ upstream putative promoter region of the ALDH1L1 locus was assessed for genomic conservation using the Vistabrowser2 [19]. $\mathrm{h} A L D H 1 L 1$ promoter was generated into MluI-BamHI restriction enzyme sites of AAV-MCS (multi cloning site) expression vector (Cell Biolabs, Inc. cat\# VPK-410) by in-Fusion cloning scheme as described previously [20]. AAV-hALDH1L1-Cre plasmid in which Cre recombinase gene expression is controlled by the $1.3 \mathrm{~kb}$ promoter was inserted into BamHI-HindIII restriction enzyme sites of AAV-hALDH1L1-MCS vector and verified by sequencing. Similar to AAV-hALDH1L1-Cre plasmid, AAV-CaMKIIa-Cre plasmid was constructed, and the CaMKIIa promoter was obtained from AAV-CaMKIIa-EGFP (Addgene \#50469). AAV-EF1aDIO-mCherry viral vector was purchased (Addgene \#47636).

\section{AAV vector development}

The viral vectors were pseudotyped, where the transgene of interest was flanked by inverted terminal repeats of the AAV2 packaged in an AAV-DJ capsid. AAV-DJ was engineered via DNA family shuffling technology which created a hybrid capsid from 8 AAV serotype. AAV-hALDH1L1-Cre vector was thereafter purified by iodixanol gradients by the KIST Virus Facility (http://virus. kist.re.kr). The production titer was $1.5 \times 10^{13}$ genome copies $/ \mathrm{ml}$ $(\mathrm{GC} / \mathrm{ml})$.

\section{Animal}

Adult (aged 8 10 weeks) male and female Ail4 mice (RCL-tdTomato; Rosa-CAG-LSL-tdTomato-WPRE::deltaNeo) in C57BL/6J 
strain were used as transgenic reporter line. Mice had free access to food and water and were kept on a 12 hours light-dark cycle. All experimental procedures described below were performed in accordance with the Institutional guidelines for experimental animal care and use of the Korea Institute of Science and Technology (KIST; Seoul, Korea).

\section{Stereotaxic injection}

Mice were anesthetized with $2 \%$ avertin $(20 \mu \mathrm{l} / \mathrm{g}$, i.p. $)$ and placed on stereotaxic apparatus (Kopf instrument, USA). AAVhALDH1L1-Cre virus was injected through 33 gauge blunt NanoFil needle (World Precision Instruments, USA), connected with $10 \mu$ Hamilton micro syringe (Hamilton, Switzerland) filled with distilled water. Syringe pump (KD Scientific, USA) was used to inject the virus with the rate of $0.2 \mu \mathrm{l}$ per minute. Needle was gently placed into target regions (thalamic VB: $-1.8 \mathrm{~mm} \mathrm{AP}, \pm 1.6$ $\mathrm{mm}$ ML, $3.75 \mathrm{~mm}(1 \mu \mathrm{l})$ and $3.25 \mathrm{~mm}(1 \mu \mathrm{l})$ from brain surface; hippocampal CA1: $-1.8 \mathrm{~mm} \mathrm{AP,} \pm 1.6 \mathrm{~mm} \mathrm{ML}, 1.6 \mathrm{~mm}(2 \mu \mathrm{l})$ from brain surface; BLA: $-1.8 \mathrm{~mm} \mathrm{AP}, \pm 3.4 \mathrm{~mm} \mathrm{ML}, 4.55 \mathrm{~mm}(2 \mu \mathrm{l})$ from brain surface; mPFC: $1.8 \mathrm{~mm} \mathrm{AP}, \pm 0.3 \mathrm{~mm} \mathrm{ML}, 1.7 \mathrm{~mm}(2$ $\mu \mathrm{l})$ from brain surface), bilaterally. Mice were sacrificed after 10 days recovery.

\section{Immunohistochemistry}

Mice were anesthetized with $2 \%$ avertin $(20 \mu \mathrm{l} / \mathrm{g}$, i.p.) and perfused with $0.1 \mathrm{M}$ phosphate buffered saline (PBS) at room temperature followed by ice-cold 4\% paraformaldehyde. Extracted brains were post-fixed in $4 \%$ paraformaldehyde at $4^{\circ} \mathrm{C}$ overnight. Post-fixed brains were incubated in $30 \%$ sucrose at $4^{\circ} \mathrm{C}$ for more than 24 hours. Coronal brain sections were prepared with $30 \mu \mathrm{m}$ thickness in cryostat microtome (Thermo Scientific). Brain sections were rinsed three times with PBS, and blocking step was performed with a blocking solution (0.3\% Triton X-100 (Sigma), $2 \%$ donkey serum (Millipore) and 2\% goat serum (Abcam) in 0.1 M PBS) for 1.5 hours. Blocking step was followed by application of a mixture of primary antibodies as follows, rabbit anti-Aldh1L1 antibody (Novus), mouse anti-NeuN antibody (Millipore). Sections were incubated overnight at $4^{\circ} \mathrm{C}$ with shaking for primary antibodies. After overnight incubation, the sections were washed three times in PBS and then incubated in corresponding fluorescent secondary antibodies for 1.5 hours. In three times wash process of secondary antibodies, they also were counterstained with 4,6'-diamidino-2-phenylindole (DAPI, Pierce) before they were mounted onto slide glass (Thermo Scientific). A series of fluorescence images were obtained with Nikon Al confocal microscope, and $30 \mu \mathrm{m} \mathrm{Z}$ stack images in 3- $\mu \mathrm{m}$ steps were processed for further analysis using NIS-Elements (Nikon) software and ImageJ (NIH) program. Reference atlases of mouse brain in the figure were obtained from Allen Brain Atlas (Reference Atlas, Version 2 (2011)) [21].

\section{Astrocyte culture and immunocytochemistry}

Primary cortical astrocytes were cultured from P0 to P3 C57BL/6N mouse pups. The cerebral cortex was dissected free meninges and softly triturating to single cell unit. Culture media was prepared as follow. Dulbecco's modified Eagle's medium (DMEM, Invitrogen) supplemented with $25 \mathrm{mM}$ glucose, $2 \mathrm{mM}$ glutamine, $10 \%$ heat-inactivated horse serum, $10 \%$ heat-inactivated fetal bovine serum and 1,000 units/ml penicillin-streptomycin. On the third day after culture, cells were rinsed with repeated gentle pipetting and the media was replaced to get rid of debris. The day before treatment of viruses, cells were transferred to coverslip $\left(1 \times 10^{4}\right.$ per coverslip) coated with $0.1 \mathrm{mg} / \mathrm{ml}$ Poly D-Lysine (PDL, Sigma). Cultured cells were maintained at $37^{\circ} \mathrm{C}$ in a humidified $5 \%$ $\mathrm{CO}_{2}$ incubator.

Three days after virus transduction, cultured astrocytes on coverslip were rinsed with PBS and fixed with 4\% paraformaldehyde for 20 minutes. After three times wash with PBS, immunocytochemistry was performed as similar manner in IHC as describe above. Briefly, blocking step was performed with a blocking solution (same with IHC, but no goat serum) for 1 hour. Blocking step was followed by application of primary antibody, rabbit anti-Aldh1L1 antibody for 2 hours, RT. After that, the cells were washed in PBS and then incubated in corresponding fluorescent secondary antibodies for 1 hour. In three times wash process of secondary antibodies, they also were counterstained with DAPI before coverslips were mounted onto slide glass.

\section{RESULTS}

\section{Analysis of hALDH1L1 promoter and construction of AAV vector}

Aldh1L1 has been shown to be a highly specific marker for astrocytes with a substantially broader pattern of astrocytic expression than the conventional astrocyte marker, GFAP [14]. We sought to develop hALDH1L1 promoter for potential clinical application. To identify the appropriate promoter region of the $\mathrm{h} A L D H 1 L 1$ gene and compare with other species, we extracted and aligned the chromosomal locus of ALDH1L1 for human, mouse, rat, cow, marmoset and rhesus monkey using the VISTAbrowser2 (Fig. 1A). We then selected the putative hALDH1L1 promoter region of $1.3 \mathrm{~Kb}$ including the upstream sequences from position -1028 to the transcription start site. The size is chosen to fit into $\mathrm{AAV}$ vector, which is known for its size limitation of about 


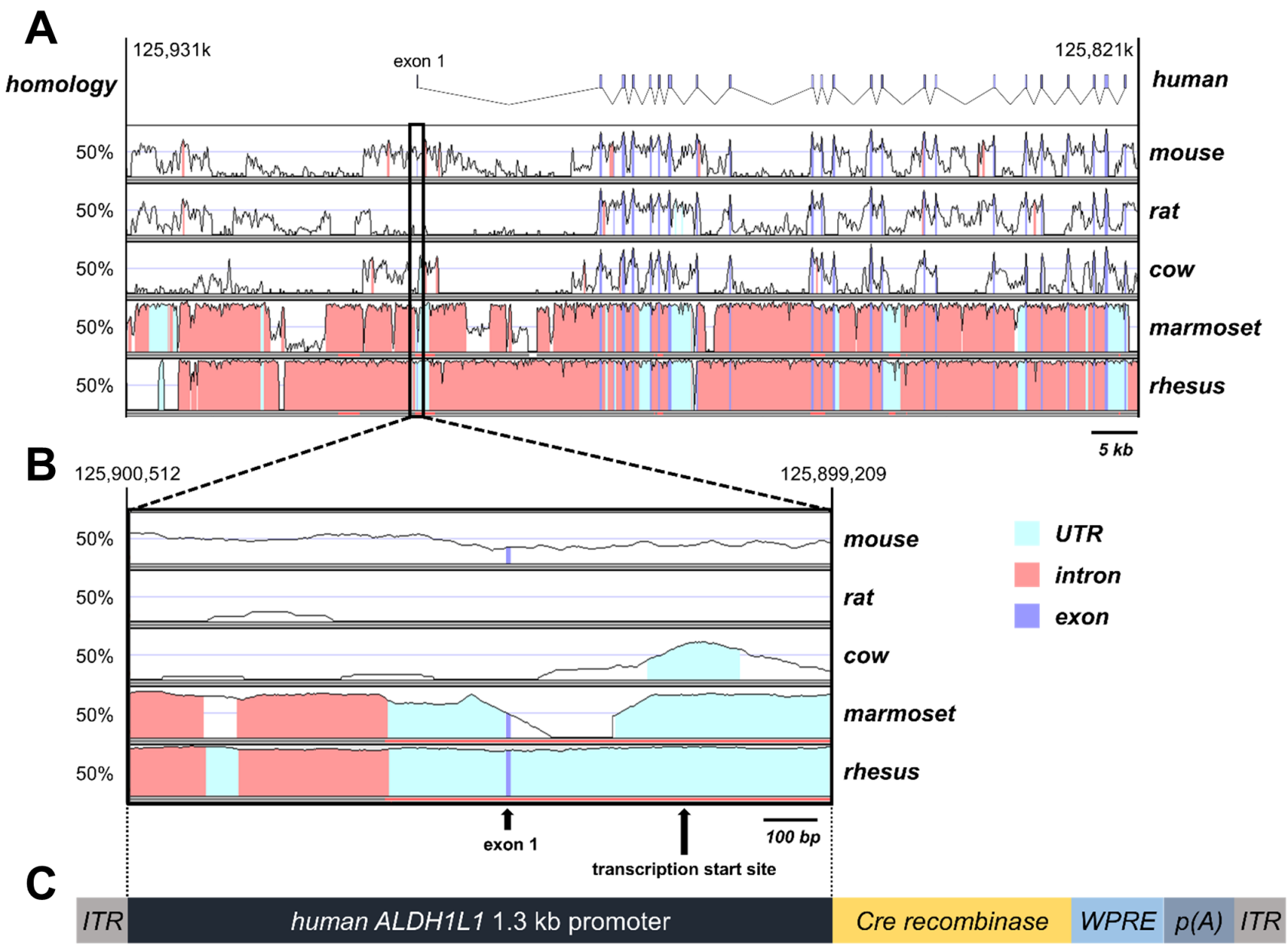

Map of AAV-hALDH1L1-Cre vector

D
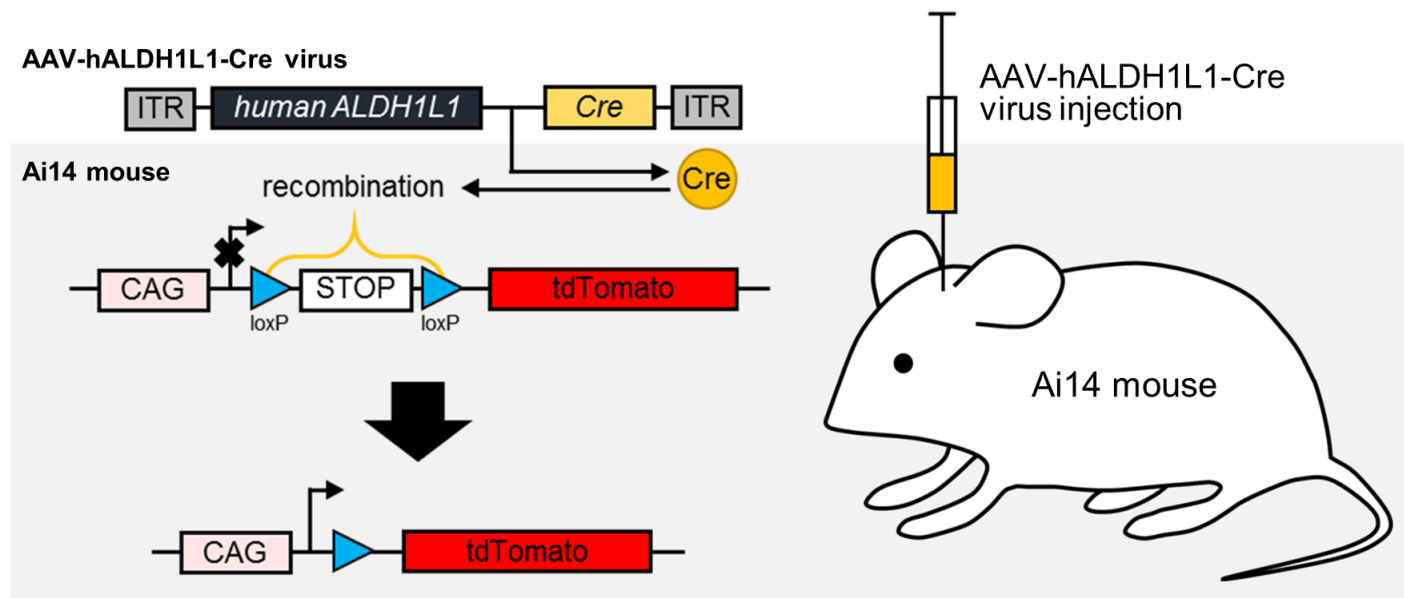

Fig. 1. ALDH1L1 promoter species alignment. (A) VISTA plot of ALDH1L1 locus from human and one from mouse, rat, cow, marmoset and rhesus monkey. (B) Expanded VISTA plot of the $1.3 \mathrm{~kb}$ upstream promoter region shows that the homology is over the $50 \%$ of mouse and other primate. And also note that exon 1 is absent in rat and cow. (C) AAV vector construction for Cre recombinase expression controlled by $1.3 \mathrm{~kb}$ hALDH1L1 promoter.(D) To investigate hALDH1L1 promoter activity, AAV-hALDH1L1-Cre virus was injected into Ail4 mouse. tdTomato protein only can be expressed when the stop codon between CAG promoter and tdTomato is eliminated by Cre recombinase which is delivered under hALDHIL1 promoter. 

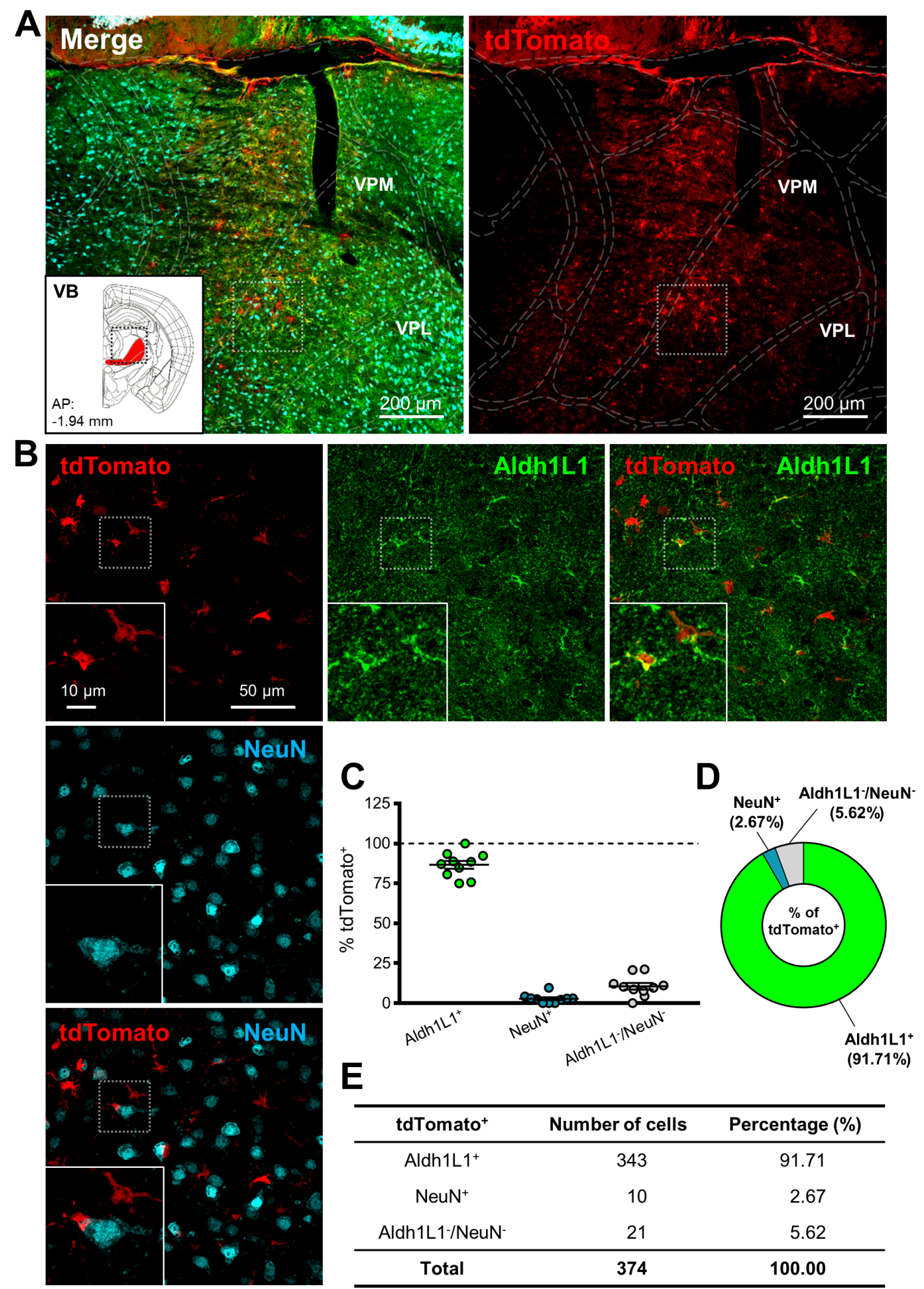

\begin{tabular}{ccc}
\hline tdTomato $^{+}$ & Number of cells & Percentage (\%) \\
\hline Aldh1L1 $^{+}$ & 343 & 91.71 \\
NeuN $^{+}$ & 10 & 2.67 \\
Aldh1L1-/NeuN & 21 & 5.62 \\
\hline Total & $\mathbf{3 7 4}$ & $\mathbf{1 0 0 . 0 0}$ \\
\hline
\end{tabular}

Fig. 2. hALDH1L1 promoter-driven Cre recombinase-mediated tdTomato expression in thalamic VB. (A) Low magnification images of injection area in thalamus. Each color represents as following description. Green indicates immunoreactivity of Aldh1L1 protein; Red indicates tdTomato expression; Cyan indicates immunoreactivity of NeuN. Inset indicates the area of target (red area, VB). (VPM, ventral posteromedial nucleus; VPL, ventral posterolateral nucleus). (B) High magnification images from the box in (A). (C) Summary graph of percentages of Aldh1L1+ (green) cells and NeuN+ (cyan) cells (and also both negative cells, gray) in tdTomato+ cells from each image (Mean \pm SEM). (D) Quantification of tdTomato+ population with Aldh1L1+, NeuN+ and Aldh1L1-/NeuN- cells in thalamic VB area. (E) Summary of tdTomato+ cells in thalamic VB area. 
$4.7 \mathrm{~Kb}$. Compared to human, mouse ALDH1L1 promoter was shown around 50\% homology of genomic conservation (Fig. 1B). In contrast, $\mathrm{r} A L D H 1 L 1$ promoter showed little homology $(<20 \%)$, suggesting that human form is a much better candidate for a use in mouse brain. Then the hALDH1L1 promoter $(1.3 \mathrm{~Kb})$ was cloned into AAV viral vector together with Cre recombinase to make AAV-hALDH1L1-Cre (Fig. 1C). Then the virus carrying AAVhALDH1L1-Cre was injected into the transgenic reporter mouse line, Ai14, which has loxP-flanked stop cassette between CAG promoter and tdTomato reporter gene (Fig. 1D). The tdTomato fluorescence is turned on when Cre recombinase is expressed by AAVhALDH1L1-Cre virus infection. Therefore, $\mathrm{h} A L D H 1 L 1$ promoter activity can be measured from the tdTomato fluorescence, and cell-type of tdTomato+ cell can be determined with immunohistochemistry.

\section{Astrocyte specific expression of hALDH1L1-Cre in thalamic VB nucleus}

To test cell-type specific expression of Cre recombinase by $\mathrm{h} A L$ DH1L1 promoter in thalamus, AAV-hALDH1L1-Cre virus was injected into thalamic VB of Ai14 mouse (Fig. 2A). To identify hALDH1L1 promoter-driven Cre recombinase-expressing cells, we performed immunohistochemistry with ALDH1L1 antibody and high magnification confocal imaging (Fig. 2B). We found that the tdTomato+ cells in VB were highly co-localized with Aldh1L1 (Fig. 2B, green, 2C). On the other hand, tdTomato+ cells in $\mathrm{VB}$ were barely co-localized with neuron-specific marker, NeuN (Neuronal nuclear antigen) (Fig. 2B, cyan, 2C). Average percentage of Aldh1L1+/tdTomato+ cells in each image was much higher than that of $\mathrm{NeuN}+/$ tdTomato+ cells (Aldh1 L1+: $86.7 \pm 2.5 \%$ versus NeuN+: $2.8 \pm 0.9 \%$ ) (Fig. $2 \mathrm{C}$ ). Among the analyzed total tdTomato+ cells, Aldh1L1+ cells were also much more than NeuN+ cells (Aldh1L1+: 91.71\% versus NeuN+: 2.67\%) (Fig. 2D and E). This result implicates that AAV-mediated $\mathrm{h} A L D H 1 L 1$ promoter-driven gene expression is highly astrocyte-specific in thalamic VB area.

\section{Mixed cell-type expression of hALDH1L1-Cre in BLA and hippocampal CAI}

Next, we tested astrocyte-specific Cre expression in amygdala and hippocampus. AAV-hALDH1L1-Cre virus was delivered to BLA and hippocampal CA1, respectively (Fig. 3A and 4A). In BLA, both cell types, astrocytes and neurons, showed tdTomato fluorescence (Fig. 3B). Average percentage of Aldh1L1+/tdTomato+ cells in each image was lower than that of $\mathrm{NeuN}+/ \mathrm{tdTomato}+$ cells (Aldh1L1+: $26.5 \pm 7.5 \%$ versus NeuN+: 69.5 $\pm 8.7 \%$ ) (Fig. 3C). Among the analyzed total Aldh1L1+ cells, tdTomato+ cells were $28.35 \%$, and NeuN+ cells were $68.13 \%$ (Fig. 3D and E). These results indi- cate that AAV-hALDH1L1-Cre virus expresses Cre recombinase in both astrocytes and neurons in BLA.

Similar to BLA, in hippocampal CA1, AAV-hALDH1L1-Cre virus induced tdTomato fluorescence from astrocytes and neurons in Ail4 mouse (Fig. 4B). Average percentage of Aldh1L1+/ tdTomato+ cells in each image was $20.4 \pm 8.8 \%$, which is lower than that of NeuN+/tdTomato+ cells (75.3 $\pm 8.6 \%)$ (Fig. 4C). In total of analyzed tdTomato+ cells in hippocampal CA1 area, a population of Aldh1L1+ cells was $18.00 \%$, and a population of NeuN+ cells was $76.25 \%$ (Fig. 4D and E). These results indicate that AAV-hALDH1L1-Cre virus expresses Cre recombinase in both astrocytes and neurons in hippocampal CA1.

\section{Neuron-specific expression of hALDH1L1-Cre in MPFC}

We further investigated activity pattern of $\mathrm{h} A L D H 1 L 1$ promoter in cortical area by injecting into mPFC (Fig. 5A). In mPFC Cremediated tdTomato expression was highly neuron-specific (Fig. $5 B)$. Average percentage of $\mathrm{NeuN}+/ \mathrm{tdTomato}+$ cells in each image was much higher than that of Aldh1L1+/tdTomato+ cells (NeuN+: $91.5 \pm 4.4 \%$ versus Aldh1L1+: 7.1 $\pm 3.8 \%$ ) (Fig. 5C). In total of analyzed tdTomato + cells in $\mathrm{mPFC}$ area, the population of $\mathrm{NeuN}+$ cells was $91.98 \%$, and the population of Aldh $1 \mathrm{~L} 1+$ cells was $6.66 \%$ (Fig. 5D and E). These results indicate that AAV-hALDH1L1-Cre is highly neuron-specific.

Taken together, expression pattern of AAV-hALDH1L1-Cre virus-mediated tdTomato fluorescence was different in different brain areas (Table 1). Additionally, we further analyzed a proportion of astrocytes that express tdTomato in these brain regions. To measure the proportion of astrocytes expressing tdTomato fluorescence, we counted all Aldh1L1+ cells near the infected areas from each brain region, and the number of tdTomato+ cells with Aldh1L1+ was divided by the total number of Aldh1L1+ cells. Calculated proportions of astrocytes expressing tdTomato were as following, VB: 81.91\%; BLA: 51.03\%; CA1: 25.55\%; mPFC: 13.26\%. These results indicate that astrocytes in VB, BLA, CA1 and $\mathrm{mPFC}$ are heterogeneous in virus transduction efficacy or $\mathrm{h} A L D H 1 L 1$ promoter activity.

As a summary, AAV-hALDH1L1-Cre virus showed highly specific expression in astrocyte of thalamic area. The virus-mediated tdTomato fluorescence could be observed in both astrocyte and neuron in amygdala and hippocampus. Finally, AAV-hALDH1L1Cre virus in $\mathrm{mPFC}$ showed highly specific expression in neuron.

\section{hALDH1L1 promoter activity in cultured system}

We further investigated the efficiency of AAV-hALDH1L1-Cre virus in cultured system. To examine the Cre recombinase expression under $\mathrm{h} A L D H 1 L 1$ promoter in primary cultured astrocyte, 


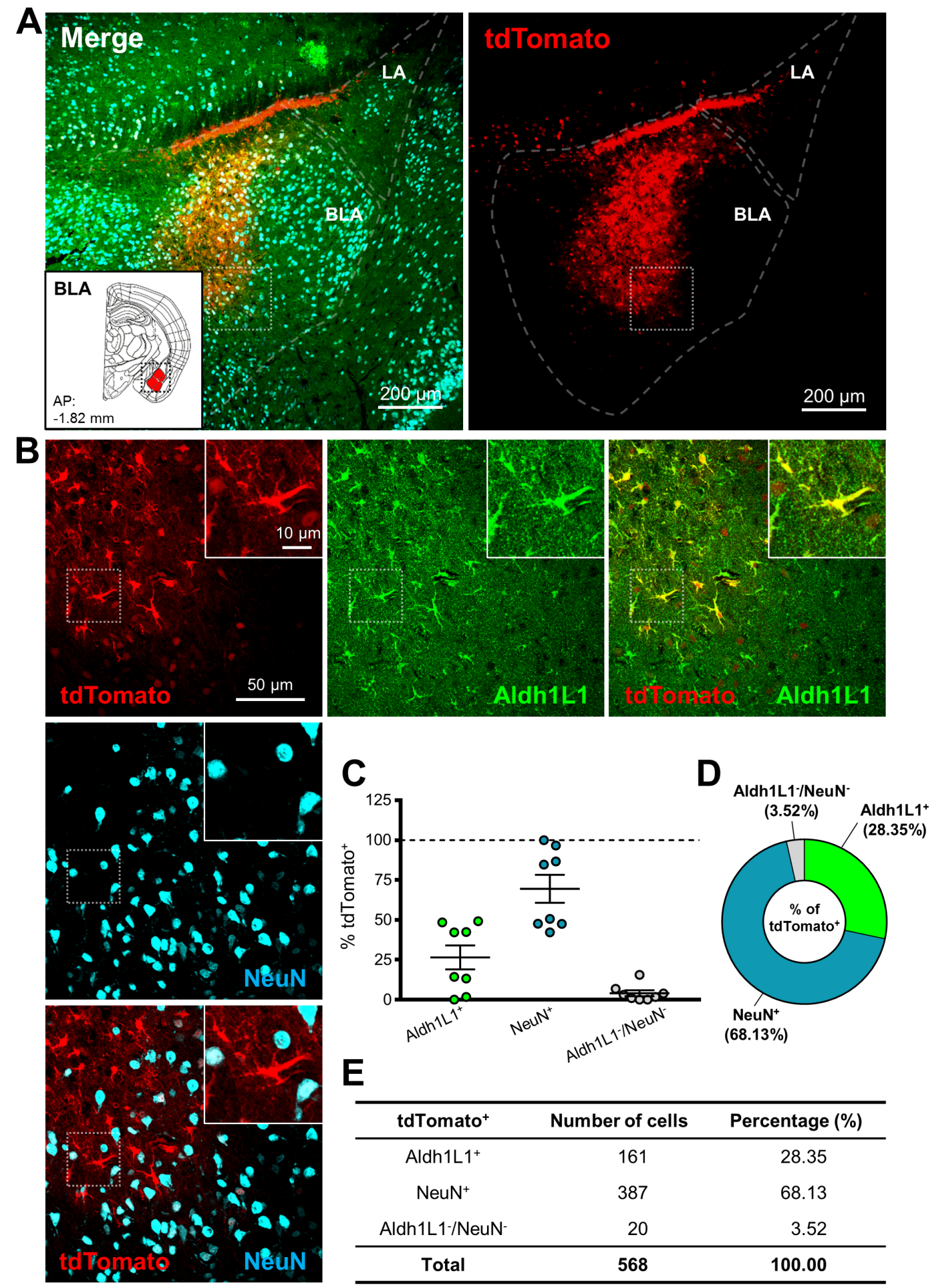

Fig. 3. hALDH1L1 promoter-driven Cre recombinase-mediated tdTomato expression in BLA. (A) Low magnification images of injection area in amygdala. Each color represents as following description. Green indicates immunoreactivity of Aldh1L1 protein; Red indicates tdTomato expression; Cyan indicates immunoreactivity of NeuN. Inset indicates the area of target (red area, BLA). (LA, lateral amygdala). (B) High magnification images from the box in (A). (C) Summary graph of percentages of Aldh1L1+ (green) cells and NeuN+ (cyan) cells (and also both negative cells, gray) in tdTomato+ cells from each image (Mean \pm SEM). (D) Quantification of tdTomato+ population with Aldh1L1+, NeuN+ and Aldh1L1-/NeuN-cells in thalamic VB area. (E) Summary of tdTomato+ cells in BLA. 

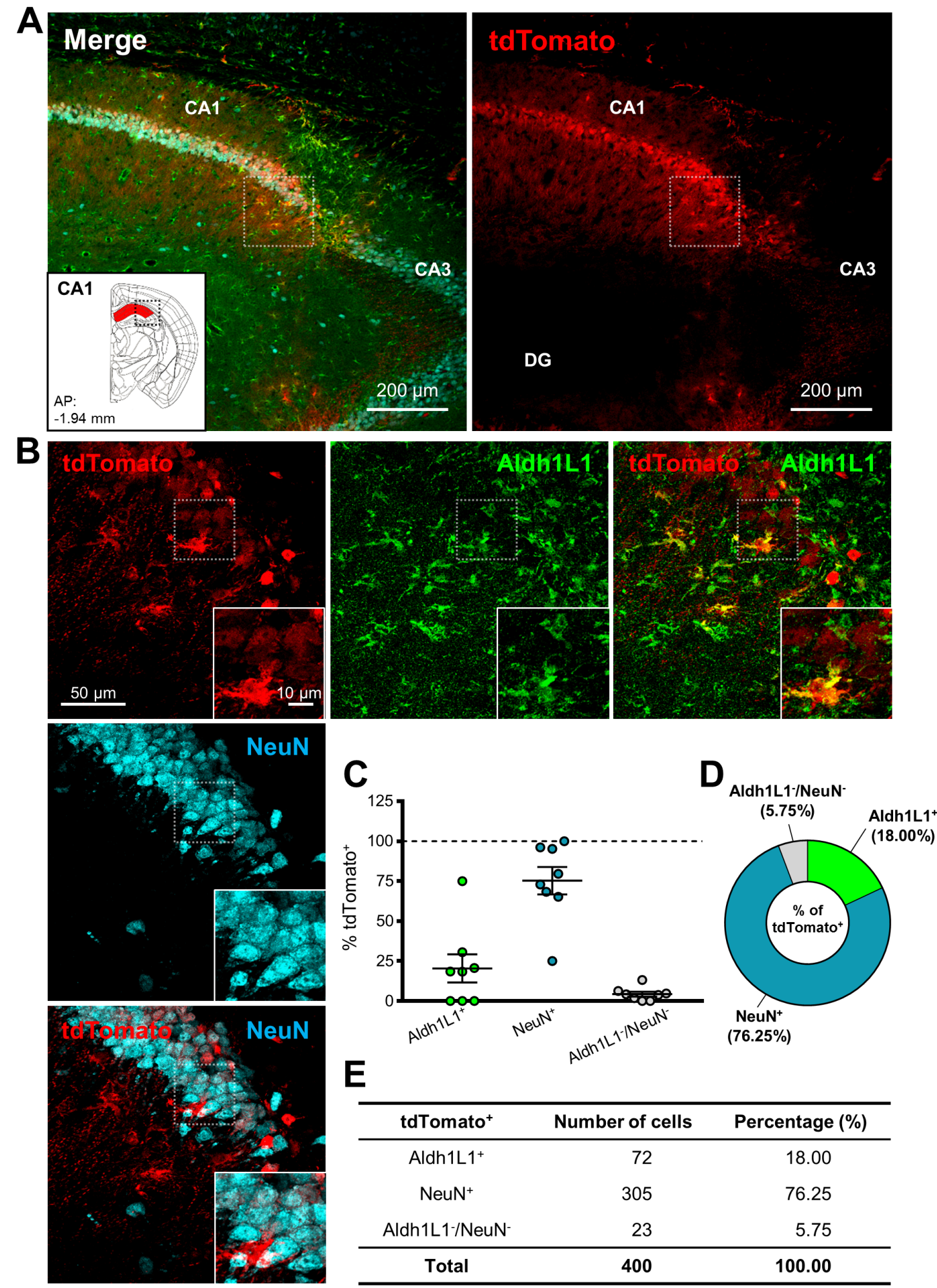

E

\begin{tabular}{ccc}
\hline tdTomato $^{+}$ & Number of cells & Percentage (\%) \\
\hline Aldh1L1+ & 72 & 18.00 \\
NeuN $^{+}$ & 305 & 76.25 \\
Aldh1L1-/NeuN- & 23 & 5.75 \\
\hline Total & $\mathbf{4 0 0}$ & $\mathbf{1 0 0 . 0 0}$ \\
\hline
\end{tabular}

Fig. 4. hALDH1L1 promoter-driven Cre recombinase-mediated tdTomato expression in hippocampal CA1. (A) Low magnification images of injection area in hippocampus. Each color represents as following description. Green indicates immunoreactivity of Aldh1L1 protein; Red indicates tdTomato expression; Cyan indicates immunoreactivity of NeuN. Inset indicates the area of target (red area, CA1). (DG, dentate gyrus). (B) High magnification images from the box in (A). (C) Summary graph of percentages of Aldh1L1+ (green) cells and NeuN+ (cyan) cells (and also both negative cells, gray) in tdTomato+ cells from each image (Mean \pm SEM). (D) Quantification of tdTomato+ population with Aldh1L1+, NeuN+ and Aldh1L1-/NeuN-cells in hippocampal CA1 area. (E) Summary of tdTomato+ cells in hippocampal CA1 area. 

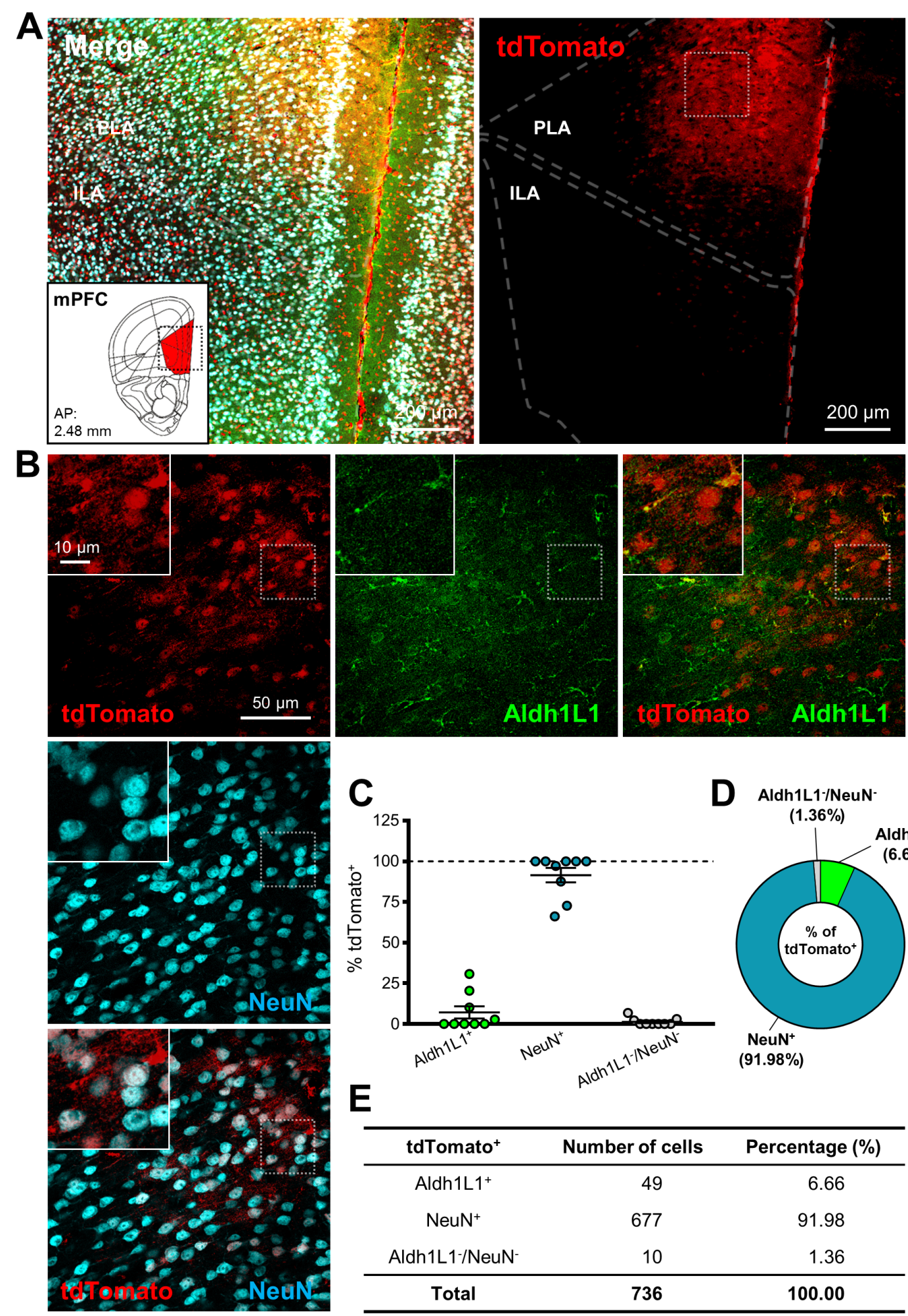

D Aldh1L1//NeuN-

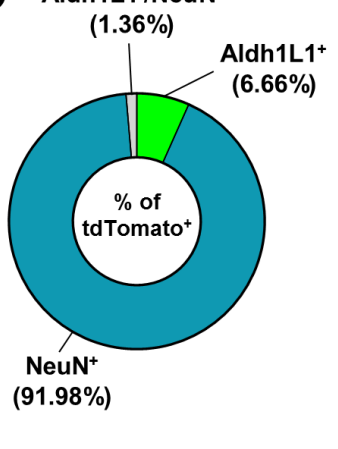

E

\begin{tabular}{ccc}
\hline tdTomato $^{+}$ & Number of cells & Percentage (\%) \\
\hline Aldh1L1 $^{+}$ & 49 & 6.66 \\
NeuN $^{+}$ & 677 & 91.98 \\
Aldh1L1-/NeuN $^{-}$ & 10 & 1.36 \\
\hline Total & $\mathbf{7 3 6}$ & $\mathbf{1 0 0 . 0 0}$ \\
\hline
\end{tabular}

Fig. 5. hALDHIL1 promoter-driven Cre recombinase-mediated tdTomato expression in mPFC. (A) Low magnification images of injection area in mPFC. Each color represents as following description. Green indicates immunoreactivity of Aldh1L1 protein; Red indicates tdTomato expression; Cyan indicates immunoreactivity of NeuN. Inset indicates the area of target (red area, mPFC). (PLA, prelimbic area; ILA, infralimbic area). (B) High magnification images from the box in (A). (C) Summary graph of percentages of Aldh1L1+ (green) cells and NeuN+ (cyan) cells (and also both negative cells, gray) in tdTomato+ cells from each image (Mean \pm SEM). (D) Quantification of tdTomato+ population with Aldh1L1+, NeuN+ and Aldh1L1-/NeuNcells in mPFC area. (E) Summary of tdTomato+ cells in mPFC area. 
Table 1. Summary of hALDH1L1 promoter activity and astrocytic transduction efficiency (\%) in four mouse brain regions, VB, BLA, CA1 and mPFC

\begin{tabular}{|c|c|c|c|c|}
\hline Cell types & $\begin{array}{c}\text { Thalamic ventrobasal } \\
\text { nucleus (VB) }\end{array}$ & $\begin{array}{c}\text { Basolateral } \\
\text { amygdala (BLA) }\end{array}$ & Hippocampal CA1 & $\begin{array}{l}\text { Medial prefrontal } \\
\text { cortex (mPFC) }\end{array}$ \\
\hline Astrocytes (\%) & 91.71 & 28.35 & 18.00 & 6.66 \\
\hline Neurons $(\%)$ & 2.67 & 68.13 & 76.25 & 91.98 \\
\hline Others (\%) & 5.61 & 3.52 & 5.75 & 1.36 \\
\hline Astrocytic transduction efficiency (\%) & 81.91 & 51.03 & 25.55 & 13.26 \\
\hline
\end{tabular}

A

AAV-hALDH1L1-Cre virus

ITR human ALDH1L1 Cre ITR

AAV-CaMKIla-Cre virus

ITR CaMKIIa $\mathrm{Cre}$ ITR

AAV-EF1a-DIO-mCherry virus

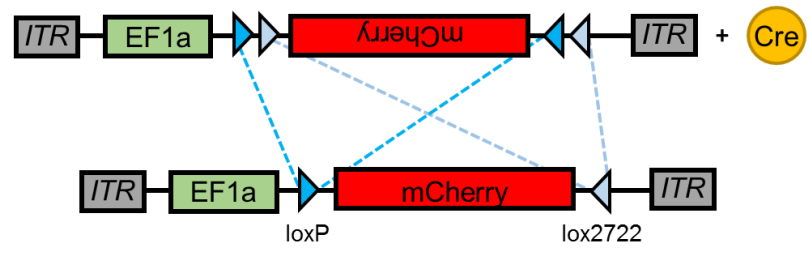

B
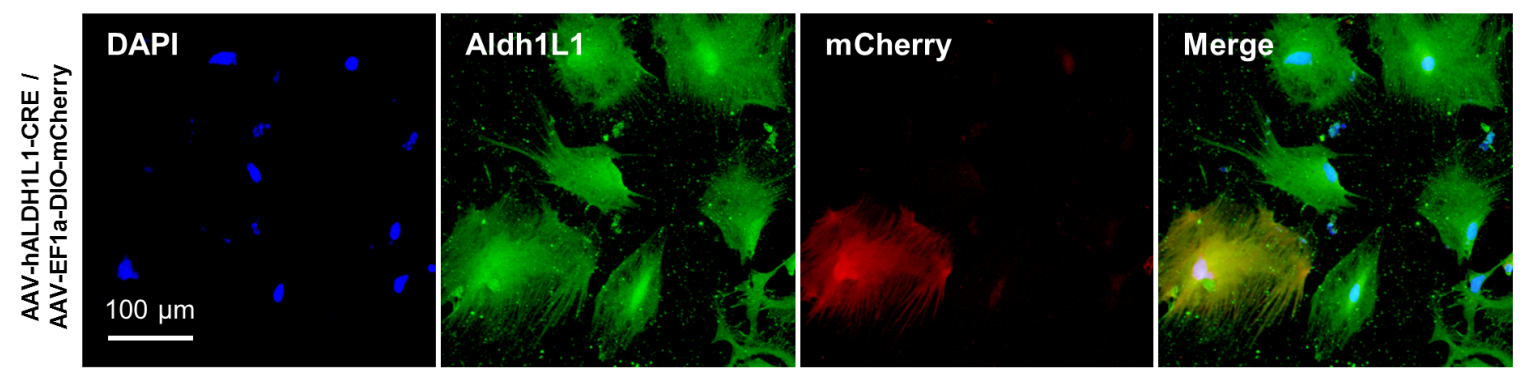

C
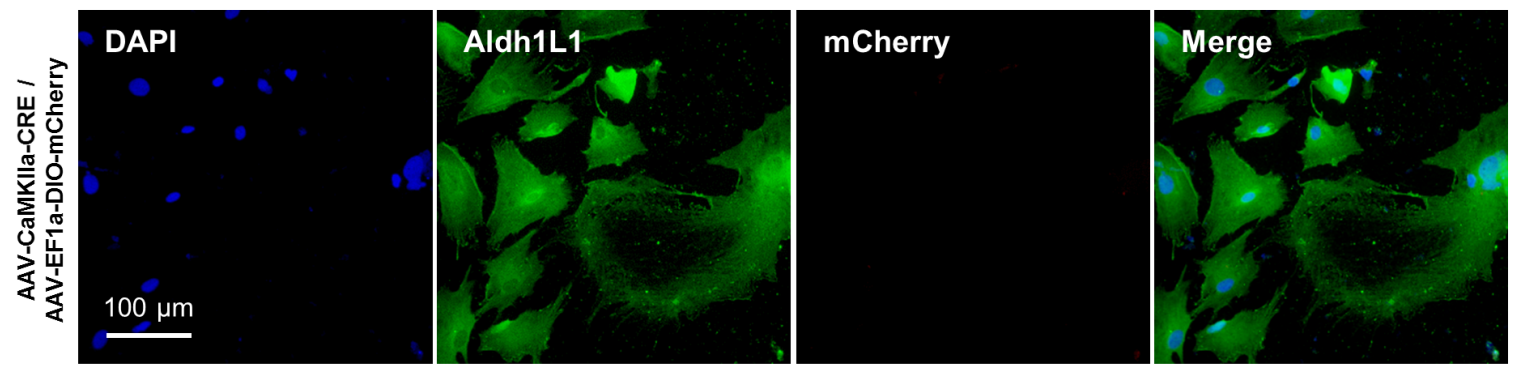

Fig. 6. AAV-hALDH1L1-Cre virus test in primary cultured system. (A) Map of AAV vectors expressing Cre recombinase under hALDH1L1 and CaMKIIa promoter. To investigate $\mathrm{h} A L D H 1 L 1$ and CaMKIIa promoter activity in naïve primary cultured cortical astrocytes, AAV-EF1a-DIO-mCherry virus was co-transduced. Reporter mCherry fluorescence protein can be expressed when a recombination occurs at Cre-loxP recombination sites (DIO: Double-floxed inverse Orientation) by Cre recombinase activity. AAV-CaMKIIa-Cre virus, whose promoter is known as excitatory neuron-specific, was used as a negative control. (B) Immunocytochemistry results showing mCherry expression by Cre recombinase in the condition of treatment with two viruses, AAV-hALDH1L1-Cre and AAV-EFla-DIO-mCherry viruses. (C) Immunocytochemistry results showing no mCherry expression in negative control condition of treatment with two viruses, AAV-CaMKIIa-Cre and AAV-EF1a-DIO-mCherry viruses. Each color represents as following description. Blue indicates nuclear staining with DAPI; Green indicates astrocyte staining with Aldh1L1; Red indicates recombinase-dependent mCherry expression.

AAV-hALDH1L1-Cre virus was treated in primary cultured cortical astrocyte. AAV-CaMKIIa-Cre virus was utilized as a negative control because the CaMKIIa promoter is specific to excitatory neuron, but not astrocyte. Due to the lack of fluorescence gene in those viruses, AAV-EF1a-DIO-mCherry virus was co-transduced as a reporter (Fig. 6A). Four days after transduction of viruses 
on cultured astrocyte, in AAV-hALDH1L1-Cre and AAV-EF1aDIO-mCherry viruses treated condition, mCherry fluorescence could be observed (Fig. 6B). However, AAV-CaMKIIa-Cre and AAV-EF1a-DIO-mCherry viruses treated condition did not show mCherry fluorescence (Fig. 6C). These results indicate that $\mathrm{h} A L$ DHIL1 promoter-mediated gene delivery for astrocyte is feasible in cultured system.

\section{DISCUSSION}

Our study is the first development and characterization of hALDH1L1 promoter. We have demonstrated that the degree of astrocyte-specific targeting by $\mathrm{h} A L D H 1 L 1$ promoter is variable in different brain regions (Table 1). There could be several reasons why expression pattern is different among different brain areas.

One possible reason is that serotype of AAV virus could affect cell-type specificity of virus infection, as many studies previously reported [22-24]. Natural AAV viruses have a specific pattern of infection that reflects the interaction and recognition between the viral capsid, envelope and receptors expressed in susceptible cells. In particular, although AAV serotype 4 and $\mathrm{AAV}$ rh 43 are known to target astrocytes $[7,25]$, the receptor of this serotype has not yet been identified. Moreover, pseudotype-dependent lentivirus has been utilized to target for astrocyte. In the current study, we utilized AAV-DJ serotype to target astrocytes with hALDH1L1 promoter. AAV-DJ serotype shows higher transduction efficiency than any natural serotype in vitro; it displays very high infectivity across a broad range of cell types in vivo [12]. In short, AAVDJ serotype is more optimized for higher transduction efficiency, regardless of cell types. Thus, the apparent differential expression pattern among brain areas may be a result of the intrinsic promoter activity rather than AAV serotype.

Another possible reason could be that hALDH1L1 promoter has less specificity in mouse brain. Although mouse ALDH1L1 promoter shows more than $50 \%$ of homology to human counterpart, the difference might be enough to influence cell-type specificity. However, as previously reported, $\mathrm{r} A L D H 1 L 1$ promoter also showed different patterns in different rat brain areas [18], which implies that even in the same species the ALDH1L1 promoter could result in different expression pattern in different brain regions. To make the hALDH1L1 more specific to astrocytes in various brain regions, it is necessary to optimize the promoter by trimming the promoter systematically, deleting potential transcription factor binding sites for neurons while leaving essential transcription factor binding sites for astrocytes. It is worthwhile to note that ALDH1L1-related transgenic mouse lines (ALDH1L1-EGFP or ALDH1L1-Cre/ERT2) were made by utilizing endogenous mouse
ALDH1L1 promoter with highly selective astrocyte-specificity in most of the brain regions $[16,17]$. Therefore, mouse ALDH1L1 promoter, which is small enough to fit into AAV viral vector, should be developed and characterized for astrocyte-specificity in mouse brain in the future.

Lastly, the neurons that expressed tdTomato fluorescence by $\mathrm{h} A L D H 1 L 1$ promoter may have alternative gene silencing system for endogenous ALDH1L1 promoter. Human ALDH1L1 promoter is already known to have extensive methylation sites (CpG island) [26]. Regarding the methylation, higher methylation level of CpG sites in ALDH1L1 promoter could lead to less expression of its protein [27], and indeed, it leads to less ALDHIL1 mRNA and protein expression in human lung adenocarcinomas [26]. Therefore, it will be interesting to investigate in the future the methylation level and epigenetic modulation in the neurons that showed tdTomato fluorescence.

In conclusion, $\mathrm{AAV}$-mediated gene expression under $\mathrm{h} A L$ DH1L1 promoter is astrocyte-specific in thalamic VB area. This hALDH1L1 promoter should be useful for investigations of astrocytic function in thalamic $\mathrm{VB}$ area.

\section{ACKNOWLEDGEMENTS}

This study was supported by KIST Institutional Grant (2E26662 to C.J.L.) and Creative Research Initiative Program (2015R1A3A2066619).

\section{REFERENCES}

1. Haydon PG, Carmignoto G (2006) Astrocyte control of synaptic transmission and neurovascular coupling. Physiol Rev 86:1009-1031.

2. Jo S, Yarishkin O, Hwang YJ, Chun YE, Park M, Woo DH, Bae JY, Kim T, Lee J, Chun H, Park HJ, Lee DY, Hong J, Kim HY, Oh SJ, Park SJ, Lee H, Yoon BE, Kim Y, Jeong Y, Shim I, Bae YC, Cho J, Kowall NW, Ryu H, Hwang E, Kim D, Lee CJ (2014) GABA from reactive astrocytes impairs memory in mouse models of Alzheimer's disease. Nat Med 20:886-896.

3. Stobart JL, Anderson CM (2013) Multifunctional role of astrocytes as gatekeepers of neuronal energy supply. Front Cell Neurosci 7:38.

4. Xie L, Kang H, Xu Q, Chen MJ, Liao Y, Thiyagarajan M, O'donnell J, Christensen DJ, Nicholson C, Iliff JJ, Takano T, Deane R, Nedergaard M (2013) Sleep drives metabolite clearance from the adult brain. Science 342:373-377.

5. Jacque CM, Vinner C, Kujas M, Raoul M, Racadot J, Baumann NA (1978) Determination of glial fibrillary acidic protein 
(GFAP) in human brain tumors. J Neurol Sci 35:147-155.

6. Kálmán M, Hajós F (1989) Distribution of glial fibrillary acidic protein (GFAP)-immunoreactive astrocytes in the rat brain. I. Forebrain. Exp Brain Res 78:147-163.

7. Liu Y, Namba T, Liu J, Suzuki R, Shioda S, Seki T (2010) Glial fibrillary acidic protein-expressing neural progenitors give rise to immature neurons via early intermediate progenitors expressing both glial fibrillary acidic protein and neuronal markers in the adult hippocampus. Neuroscience 166:241251.

8. Nolte C, Matyash M, Pivneva T, Schipke CG, Ohlemeyer C, Hanisch UK, Kirchhoff F, Kettenmann H (2001) GFAP promoter-controlled EGFP-expressing transgenic mice: a tool to visualize astrocytes and astrogliosis in living brain tissue. Glia 33:72-86.

9. Hirrlinger PG, Scheller A, Braun C, Hirrlinger J, Kirchhoff F (2006) Temporal control of gene recombination in astrocytes by transgenic expression of the tamoxifen-inducible DNA recombinase variant CreERT2. Glia 54:11-20.

10. Shinohara Y, Konno A, Takahashi N, Matsuzaki Y, Kishi S, Hirai H (2016) Viral vector-based dissection of marmoset GFAP promoter in mouse and marmoset brains. PLoS One 11:e0162023.

11. Almad AA, Maragakis NJ (2012) Glia: an emerging target for neurological disease therapy. Stem Cell Res Ther 3:37.

12. Lakhan R, Baylink DJ, Lau KH, Tang X, Sheng MH, Rundle CH, Qin X (2015) Local administration of AAV-DJ pseudoserotype expressing COX2 provided early onset of transgene expression and promoted bone fracture healing in mice. Gene Ther 22:721-728.

13. Daya S, Berns KI (2008) Gene therapy using adeno-associated virus vectors. Clin Microbiol Rev 21:583-593.

14. Ortolano S, Spuch C, Navarro C (2012) Present and future of adeno associated virus based gene therapy approaches. Recent Pat Endocr Metab Immune Drug Discov 6:47-66.

15. Cahoy JD, Emery B, Kaushal A, Foo LC, Zamanian JL, Christopherson KS, Xing Y, Lubischer JL, Krieg PA, Krupenko SA, Thompson WJ, Barres BA (2008) A transcriptome database for astrocytes, neurons, and oligodendrocytes: a new resource for understanding brain development and function. J Neurosci 28:264-278.

16. Heintz N (2004) Gene expression nervous system atlas (GENSAT). Nat Neurosci 7:483.
17. Srinivasan R, Lu TY, Chai H, Xu J, Huang BS, Golshani P, Coppola G, Khakh BS (2016) New transgenic mouse lines for selectively targeting astrocytes and studying calcium signals in astrocyte processes in situ and in vivo. Neuron 92:11811195.

18. Mudannayake JM, Mouravlev A, Fong DM, Young D (2016) Transcriptional activity of novel ALDH1L1 promoters in the rat brain following AAV vector-mediated gene transfer. Mol Ther Methods Clin Dev 3:16075.

19. Thoms JA, Birger Y, Foster S, Knezevic K, Kirschenbaum Y, Chandrakanthan V, Jonquieres G, Spensberger D, Wong JW, Oram SH, Kinston SJ, Groner Y, Lock R, MacKenzie KL, Göttgens B, Izraeli S, Pimanda JE (2011) ERG promotes T-acute lymphoblastic leukemia and is transcriptionally regulated in leukemic cells by a stem cell enhancer. Blood 117:7079-7089.

20. Park J, Throop AL, LaBaer J (2015) Site-specific recombinational cloning using gateway and in-fusion cloning schemes. Curr Protoc Mol Biol 110:3.20.1-3.20.23.

21. Dong HW (2008) The Allen reference atlas: a digital color brain atlas of the $\mathrm{C} 57 \mathrm{Bl} / 6 \mathrm{~J}$ male mouse. Wiley, Chichester.

22. Merienne N, Le Douce J, Faivre E, Déglon N, Bonvento G (2013) Efficient gene delivery and selective transduction of astrocytes in the mammalian brain using viral vectors. Front Cell Neurosci 7:106.

23. Pignataro D, Sucunza D, Vanrell L, Lopez-Franco E, DopesoReyes IG, Vales A, Hommel M, Rico AJ, Lanciego JL, Gonzalez-Aseguinolaza G (2017) Adeno-associated viral vectors serotype 8 for cell-specific delivery of therapeutic genes in the central nervous system. Front Neuroanat 11:2.

24. Royo NC, Vandenberghe LH, Ma JY, Hauspurg A, Yu L, Maronski M, Johnston J, Dichter MA, Wilson JM, Watson DJ (2008) Specific AAV serotypes stably transduce primary hippocampal and cortical cultures with high efficiency and low toxicity. Brain Res 1190:15-22.

25. Lawlor PA, Bland RJ, Mouravlev A, Young D, During MJ (2009) Efficient gene delivery and selective transduction of glial cells in the mammalian brain by AAV serotypes isolated from nonhuman primates. Mol Ther 17:1692-1702.

26. Oleinik NV, Krupenko NI, Krupenko SA (2011) Epigenetic silencing of ALDH1L1, a metabolic regulator of cellular proliferation, in cancers. Genes Cancer 2:130-139.

27. Deaton AM, Bird A (2011) CpG islands and the regulation of transcription. Genes Dev 25:1010-1022. 